\title{
Physical Activity and Mental Health in a Student Population.
}

\author{
Philip Tyson $^{1}$, Kelly Wilson ${ }^{1}$, Diane Crone ${ }^{1}$, Richard Brailsford ${ }^{1} \&$ Keith Laws ${ }^{2}$ \\ ${ }^{1}$ University of Gloucestershire, U.K. \\ ${ }^{2}$ University of Hertfordshire, U.K.
}

Corresponding Author;

Dr Philip John Tyson

School of Natural and Social Sciences

University of Gloucestershire

Francis Close Hall Campus

Swindon Road

Cheltenham

Gloucestershire GL50 4AZ, UK

Email: ptyson@glos.ac.uk

Tel: 01242714755 


\title{
Physical Activity and Mental Health in a Student Population.
}

\begin{abstract}
Background

A growing body of literature indicates that physical activity can have beneficial effects on mental health. However, previous research has mainly focussed on clinical populations, and limited research has been conducted on the psychological effects of physical activity in those without clinically defined disorders.
\end{abstract}

AIMS

The present study investigates the association between physical activity and mental health in an undergraduate university population based in the United Kingdom.

Method

One hundred students completed questionnaires measuring their levels of anxiety and depression using the Hospital Anxiety and Depression Scale (HADS) and their physical activity regime using the Physical Activity Questionnaire (PAQ).

Results

Significant differences were observed between the low, medium and high physical activity groups on the mental health scales, indicating better mental health for those who engage in more physical activity.

Conclusions

Engagement in physical activity can be an important contributory factor in the mental health of undergraduate students.

Declaration of Interest: None 
Introduction

The positive relationship between physical activity and health has been well documented (see for example Blair et al., 2001; Department of Health, 2004). Furthermore, the positive alliance between physical activity, mental health and the treatment of mental health problems, has also been demonstrated, culminating in numerous papers and reviews (for example Biddle et al., 2000; Fontaine, 2000; Lawler and Hopker, 2001; Phillips et al., 2003; Dunn et al., 2005; Penedo and Dahn, 2005; Saxena et al., 2005; Stathopoulou et al., 2006). This has resulted in the promotion of physical activity, within health policy and practice, for the improvement of both physical and mental health in the general population.

In terms of the relationship between physical activity and mental health problems, the majority of the evidence exists with regard to depression and anxiety related disorders. In respect to depression, although the range of measures used to define depression makes the comparison between studies problematic, there is a consensus that there is a negative link (Mutrie, 2000). According to Crone et al., (in press) reviews generally conclude that physical activity has a beneficial effect on mild to moderate depression (Lawler and Hopker, 2001; Craft \& Perna, 2004; Faulkner and Biddle, 2004; Craft, 2005) and public health recommendations for aerobic physical activity have been found to be an effective treatment for mild to moderate major depressive disorder (Dunn et al., 2005). Research on the effect of physical activity on anxiety has included a number of recent meta-analysis and systematic reviews, for example Long and van Stavel (1995) and Hamer et al., (2005). These too have concluded a negative relationship, particularly for aerobic exercise, on anxiety related disorders. Furthermore, Biddle and Mutrie (2001) argue that the evidence gathered to date suggests that physical activity, and in particular exercise, can be as effective at alleviating anxiety as any other medication free treatment.

Although there has been a known relationship between physical activity and mental health for some time, there still remains a lack of consensus regarding which mechanism is responsible (Crone et al., 2005; 2006). It is thought likely that this is a combination of physiological, biochemical and psycho-social aspects (Biddle and Mutrie, 2001), however, there is also 
growing evidence that the actual process of engaging in physical activity (e.g., in providing distraction from symptoms), rather than the activity itself, is influential in eliciting various mental health benefits (Faulkner and Sparkes, 1999). Furthermore, involvement in certain physical activities has been found to have other holistic benefits, for example the opportunity for social interaction (Crone et al. 2005), psycho-social benefits such as positive emotional experiences (Carless and Douglas, 2004; Carless and Sparkes, 2008; Crone, 2007), a sense of achievement (Fogarty and Happell, 2005) and improvements in general mental wellbeing (La Forge et al., 2002).

Regardless of whether the relationship between physical activity and mental health is fully understood, previous research does conclude that physical activity does have beneficial effects on general mental health. Within the Higher Education environment, it is surprising that little research has explored the potential benefits of physical activity on a cohort of individuals who are at risk of mental health problems, i.e., University students. Of the few studies that have been conducted, Ahmadi et al. (2002) reported that engaging in body building and swimming reduced scores on the Beck Depression Inventory in female students, whilst Toscovic (2001) found that students engaging in dynamic Taekwondo also reported lowers of levels of depression than a control group. Research into the mental health and wellbeing of students is particularly important as some studies have identified that anxiety and depression are a common problem, with students likely to suffer mental health problems because of concerns regarding their studies (Wardle et al., 2004). Other concerns may include tuition fees and moving away from home. Furthermore, Harrison (1999) concluded that students were 1.64 times more likely to experience symptoms of mental ill health than other young people. Worryingly, one study reported that $60 \%$ of undergraduate students had elevated levels of anxiety and depression (Inam et al., 2003). As a consequence of the findings from this limited amount of research with a university population, and of the increasing numbers of young people attending university in the United Kingdom, this study aimed to investigate self-reported levels of anxiety and depression and compare these with self-reported physical activity behaviour, in a UK based University. 
Method

\section{$\underline{\text { Participants }}$}

One hundred undergraduate university students (male $=20$; female $=80$ ) were recruited via opportunity sampling from the Faculty of Education, Humanities and Sciences at the University of Gloucestershire. Ninety percent were traditional age students (mean age 20.4 years) whilst ten percent were older (mean age 35 years). All spoke English as their first language and none had any physical impairment which would have hindered their ability to engage in physical activity.

\section{Materials}

Hospital Anxiety and Depression Scale (HADS)

The HADS (Zigmond \& Snaith, 1983) is a fourteen item self-report questionnaire with seven questions relating to anxiety (e.g., I get sudden feelings of panic) and seven questions relating to depression (e.g., I feel as if I am slowed down). Scoring is based on a 4 point likert scale $(0=$ Not at all; $1=$ Not very much; $2=$ Quite a lot; $3=$ Definitely). The maximum score for each subscale is 21 . For both subscales, scores of 0-7 indicate the absence of clinical symptoms of anxiety and depression, scores of 8-10 indicate mild symptoms, 11-14 moderate symptoms and $15-21$ severe symptoms. This scale has good psychometric properties in terms of factor structure, inter-correlation and internal consistency (Mykletun et al. 2001). The scale was originally designed to detect elevated levels of anxiety and depression amongst patients in non-psychiatric hospital clinics, and therefore is suitable to use with any population who do not have a clinically defined psychiatric disorder.

Physical Activity Questionnaire (PAQ)

The PAQ (Thirlaway \& Benton, 1992) was designed to investigate the effects of physical activity and cardiovascular fitness on mental health and mood and is based on the assumption that the more engagement someone has with aerobic exercises, the more beneficial this will 
be. Participants are required to record the details of the physical activity they undertook during the previous four weeks, in terms of type (e.g. running, swimming), frequency (number of times per week) and duration of activity. The total duration of physical activity, per month, was calculated to the nearest minute. In line with Thirlaway and Benton (1992) each type of exercise is then assigned a numerical value which reflects estimated oxygen uptake per minute (estimates based on Cooper, 1970). Strenuous activities (e.g., running, cycling) are assigned a higher numerical value than more sedentary activities (walking, Yoga). The total time spent on an activity is then multiplied by this value to give a total physical activity score.

Results

\section{Depression and Anxiety}

The mean anxiety score for the student cohort was 8.5 (S.D. = 3.9; range 1-19). This falls within the mild symptom range. For depression, the mean score was 3.6. (S.D. = 2.7; range $0-14)$. This falls within the normal range of depressive classification. Considering the percentage of students who fall within the different severity band ratings of anxiety and depression, $40 \%$ of students were considered normal on the anxiety subscale and $90 \%$ on the depression subscale. Only $6 \%$ of students were considered to have severe anxiety, with no students falling into this severity range for depression. See Table 1.

\section{Insert Table 1 about here}

\section{Physical Activity}

Participants were separated into three non overlapping groups based on their physical activity score to enable comparisons to be made between physical activity levels and scores on the HADS. See Table 2. The three groups did not differ in age ( $F=1.6, p=.20)$.

\section{Insert Table 2 about here}




\section{Associations between physical activity and mental health}

A one-way ANOVA revealed a significant main effect for depression $(F=11.34, p=<0.001$, eta $=.19)$. Post hoc $t$-tests revealed that the high and low group and the high and medium physical activity groups differed significantly (both $p$ 's $=<0.001$ ). The medium and low physical activity groups did not differ significantly $(p=>0.05)$. For anxiety, a one-way ANOVA revealed a significant main effect $(F=43.1, p=<0.001$, eta $=.47)$. Post hoc $t$-tests revealed significant differences between each physical activity group (all $p$ 's $<0.05$ ).

\section{Insert figure 1 about here}

\section{Correlational Analysis}

Scores on the depression subscale of the HADS and PAQ showed a significant negative correlation ( $r=-0.607, n=100, p=0.01$, two-tailed). A similar effect was observed for the anxiety subscale of the HADS ( $r=-0.588, n=100, p=0.01$, two-tailed). The effect sizes for the two analyses were $d=1.5$ and $d=1.4$, respectively.

\section{Discussion}

The purpose of the present study was to examine the relationship between anxiety and depression and physical activity in an undergraduate student population. Results revealed that students who engaged in high levels of physical activity showed significantly lower levels of anxiety and depression than the medium and low physical activity groups. The results from these comparisons are confirmed by the correlational analysis. Treating our cohort as a whole, we found that increased amounts of physical activity were negatively correlated with 
self-reported anxiety and depression. That is to say, as physical activity levels increased, self reported levels of anxiety and depression decreased. These findings are consistent with two other studies in this area (Ahmadi et al. 2002; Toskovic, 2001), whilst not being consistent with a third (Mack et al. 2000).

Although the findings of this study are nothing new in that there is a well established literature which shows that physical activity has beneficial consequences for mental health (Biddle et al., 2000; Dunn et al., 2005; Penedo and Dahn, 2005; Saxena et al., 2005; Stathopoulou et al., 2006), this study is one of the few which has explored this issue in a population of University students. Furthermore, most previous studies have considered this issue with clinically defined populations, this study utilised a cohort of participants who were not primarily identified as having any mental health problems.

This study suggests a dose-response relationship between physical activity and mental health, the greater the physical activity the lesser the symptoms of anxiety and depression. However, the issue of the dose -response relationship between physical activity and mental health is an unresolved one. Although many studies have suggested that greater amounts of physical activity are associated with a reduction in depression (e.g., Hassmen et al. 2000) and anxiety (e.g., Steptoe et al. 1989), a review by Dunn et al. (2001) indicates that most of these studies have been either observational or quasi-experimental in nature with no randomised controlled trials having being conducted. Therefore this relationship, although plausible, remains yet to be convincing.

Furthermore, while these results suggest a relationship between greater physical activity and lower levels of depression and anxiety, they do not infer a cause and effect relationship. That is, it is difficult to say that higher levels of depression and anxiety are due to a lack of physical activity. It may be the case that the more depressed and anxious an individual becomes, the less likely they are to engage in physical activity. This suggestion is certainly supported in the case of depression, as depressed people are less likely to want to engage in physical activities (Ussher et al. 2007; Paluska and Schwenk , 2000) and a common symptom of 
depression is a loss of interest in regular activities and psychomotor retardation (DSM-IV-TR; APA, 1994). However, our findings of a very low incidence of depression in our student population does not suggest that these symptoms are the cause of a lack of engagement in physical activity. In terms of anxiety, our results in this study suggest that, by engaging in physical activity, individuals may be able to manage levels of anxiety. However, again, increased apprehension and worry may serve to inhibit a person, and prevent them from, for example, attending a gymnasium or exercise class. Our findings of elevated levels of anxiety in our population does suggest that this may be a contributory factor in the lack of engagement in physical activity, although it is also important to note that anxiety comes in many forms, only some of which (e.g. social phobia) may inhibit a student's engagement.

Previous studies which have looked at the mental health of University students have suggested an increased risk of problems (Wardle et al., 2004; Harrison, 1999; Inam et al. 2003). The current study found that, in terms of anxiety, $60 \%$ of students showed elevated levels which does confirm previous findings. Students are faced with multiple stressors (academic work, employment, finances, housing, relationships ect) and so these findings are perhaps not surprising. In terms of depression, only $10 \%$ of students showed elevated levels which indicates that this problem is much less prevalent. Indeed, no students were reported to be in the severe category for depression. Certainly though, the issue of potential mental health problems in University students is one which needs to be proactively and continually monitored by academic institutions.

While the present study has revealed a positive link between increased physical activity and mental health promotion, limitations are present. Firstly, the issue of cause and effect requires a more specific analysis. Longitudinal randomised controlled trials are certainly needed to help answer this question. Secondly, as North et al (1990) suggest, the precise factors affecting the lower levels of depression and anxiety in the high physical activity group are important to consider. It remains unresolved whether the differences stem from physiological, biochemical or psycho-social means (Biddle and Mutrie, 2001; Crone et al., 2005), and a clarification of the mechanism of action should be one of the goals of future research. 
Furthermore, qualitative research into the perceptions of the role of physical activity in the lives of students for the maintenance of good mental health may also shed light on this relationship. Indeed, qualitative methodology is already making an important contribution to this literature, for people with mental health problems (e.g., Crone \& Guy, 2008). Future research on this topic should also consider whether there are different prevalence rates of mental health problems amongst male and female students, and if physical activity affects the mental health of male and female students differently. The current study was limited by having an overrepresentation of female participants.

Finally, our findings contribute to the growing body of literature demonstrating the positive effects of physical activity on mental health. Conclusions should be of interest to students wishing to maintain and promote their mental health while at university, and for universities wishing to safeguard their students emotional well-being through the promotion of physical activity. 
References

Ahmadi, J., Samavat, F., Sayyad, M. \& Ghanizadeh, A. (2002) Various types of exercise and scores on the Beck Depression Inventory. Psychological Reports, 90 (3), 821-822.

American Psychiatric Association (2000) Diagnostic and Statistical Manual of Mental

Disorders. Washington D.C. : American Psychiatric Press.

Bernstein, L., Henderson, B.E., Hanisch, R., Sullivan-Halley, J. and Ross, R.K. (1994)

Physical exercise and reduced risk of breast cancer in young women. Journal of the National Cancer Institute, 86(18), 1403-1408.

Biddle, S.J.H., Fox, K.R. \& Boucher, S.H. (2000). Physical Activity and Psychological WellBeing. London: Routledge.

Biddle, S.J.H. \& Mutrie, N. (2001) Psychology of Physical Activity; Determinants, Well-Being and Interventions. London: Routledge.

Blair, S., Cheng, Y. \& Scott Holder, J. (2001), Is physical activity or physical fitness more important in defining health benefits? Medicine and Science in Sports and Exercise, 33(6), S379 - S399.

Callaghan, P. (2004) Exercise: a neglected intervention in mental health care? Journal of Psychiatric and Mental Health Nursing, 11, 476-483.

Cooper, K.H. (1970) The New Aerobics. New York: Bantum Books.

Craft, L.L. \& Perna, F.M. (2004) The Benefits of Exercise for the Clinically Depressed, Primary Care Companion, Journal of Clinical Psychiatry, 6, $104-111$. 
Carless, D. \& Douglas, K. (2004). A golf programme for people with severe and enduring mental illness. Journal of Mental Health Promotion, 3(4), 26-39.

Carless, D. \& Sparkes, A. (2008). Narrative, identity and mental health: How men with serious mental illness re-story their lives through sport and exercise. Psychology of Sport and Exercise, 9 (5), 576-594

Craft, L. (2005). Exercise and clinical depression: examining two psychological mechanisms. Psychology of Sport and Exercise, 6 (2) 151-171.

Crone, D. (2007) Walking back to health: A qualitative investigation into service user's experiences of a walking project. Issues in Mental Health Nursing, 28 (2), 167-183.

Crone, D. \& Guy, H. (2008) 'I know it is only exercise, but to me it is something that keeps me going'. A qualitative approach to understanding mental health service users' experiences of sports therapy. International Journal of Mental Health Nursing, 17, 197-207.

Crone, D., Smith, A. \& Gough, B. (2005) 'I feel totally alive, totally happy and totally at one': A psycho-social explanation of the physical activity and mental health relationship from the experiences of participants on exercise referral schemes. Health Education Research, 20 (5), $600-611$.

Department of Health (2004). At least five a week. Evidence of the impact of Physical Activity and It's Relationship to Health. A report from the Chief Medical Officer. London: HMSO.

DiLorenzo, T.M., Bargman, E.P., Stucky-Ropp, R., Brassington, G.S., Frensch, P.A. \& LaFontaine, T. (1999) Long-term effects of aerobic exercise on psychological outcomes. Preventive Medicine, 28, 75-85. 
Dunn, A.L., Trivedi, M.H. and O'Neal, H.A. (2001) Physical Activity dose-response effects on outcomes of depression and anxiety. Medicine \& Science in Sports \& Exercise, 33, (6 Suppl) :S587-97.

Dunn , M . Trivedi , J . Kampert , C . Clark , H . Chambliss (2005). Exercise treatment for depression Efficacy and dose response. American Journal of Preventive Medicine, 28 (1), $1-8$.

Edelmann, R. (1995). Anxiety: Research, Theory and Intervention in Clinical and Health Psychology. Chichester: John Wiley.

Faulkner, G., Biddle, S. J. H. (2004). Exercise and depression: considering variability and contextuality. Journal of Sport \& Exercise Psychology, 26 (1) 3-18.

Faulkner, G. \& Sparkes, A. (1999) Exercise as therapy for schizophrenia: An ethnographic study. Journal of Sport and Exercise Psychology, 21, 52-69.

Fogarty, M., \& Happell, B. (2005). Exploring the benefits of an exercise program for people with schizophrenia: A qualitative study. Issues in Mental Health Nursing, 26, 341-351.

Fontaine, K.R. (2000). Physical activity improves mental health. The Physician and Sports Medicine, 28 (10), 83-84.

Hamer, M., Taylor, A. \& Steptoe, A. (2005). The effect of acute aerobic exercise on stress related blood pressure responses: a systematic review and meta-analysis. Biological Psychology, 71, $183-190$.

Harrison, L., (1999). Student Mental Health, [Online] Available at http/: www.brookes.ac.uk. [Accessed on 14/11/2007]. 
Hassmen, P., Koivula, N. and Uutela, A. (2000) Physical exercise and psychological well being: a population study in Finland. Preventative Medicine, 30: 17-25, 2000.

Inam, S.N., Saqib, A. \& Alam, E. (2003). Prevalence of anxiety and depression among medical students of a private university. Journal of the Pakistani Medical Association, 53 (2), 44-7.

Lawler D, Hopker S. (2001). The effectiveness of exercise as an intervention in the management of depression. British Medical Journal ; 322: 1-8.

Long, B.C \& van Stavel, R. (1995). Effects of exercise training on anxiety: A meta-analysis. Journal of Applied Sport Psychology, 7(2), 167 - 189.

Mack, M.G., Huddleston, S., Dutler, K.E. \& Bian, W. (2000) Mood state changes of students enrolled in physical activity classes. Perceptual \& Motor Skills, 90 (3), 911-914.

Mutrie, N. (2000) The relationship between physical activity and clinically defined depression. In S.J.H. Biddle, K.R. Fox, \& S.H. Boutcher (Eds.) Physical Activity and Psychological Wellbeing (pp. 46-62) London. Routledge.

Mykletun, A. , Stordal, E. and Dahl, A. (2001) Hospital Anxiety and Depression (HAD) Scale: factor structure, item analyses and internal consistency in a large population. British Journal of Psychiatry, 179, 540-544.

North, T.C., McCullagh, P. and Tran, Z.V. (1990) Effect of exercise on depression. In: K.P Pandolf \& J.O Holloszy (Eds.) Exercise and Sport Science Reviews. (pp. 379-415). Baltimore, Williams and Wilkins.

Laforge, R.G., Rossi J.S., Prochaska J.O., Velicer, W.F. Levesque, D.A. and McHorney C.A. (2002) Stage of Exercise and Health Related Quality of Life. Journal of Preventive Medicine, 28(4) $349-360$. 
Paluska, S.A. and Schwenk, T.L. (2000) Physical activity and mental health: current concepts. Sports Medicine, 29(3), 167-180.

Penedo, F.J. \& Dahn, J.R. (2005). Exercise and well-being: a review of mental and physical health benefits associated with physical activity. Current Opinion in Psychiatry. 18(2):189193.

Riebe, D., Greene, G.W., Ruggiero, L., Stillwell, K.M., Blissmer, B., Nigg, C.R. and Caldwell, M. (2003) Evaluation of a healthy lifestyle approach to weight management. Preventive Medicine, 36(1), 45-54.

Saxena, S., Van Ommeren, M., Tang, K. \& Armstrong, T. (2005) Mental health benefits of physical activity. Journal of Mental Health, 14 (5), 445-451.

Stathopoulou, G., Powers, M.B., Berry, A.C., Smits, J.A.J. \& Otto, M.W. (2006). Exercise Interventions for Mental Health: A Quantitative and Qualitative Review, Clinical Psychology: Science and Practice 13 (2), 179-193.

Steptoe, A., Edwards, S., Moses, J. and Mathews, A. (1989) The effects of exercise training on mood and perceived coping ability in anxious adults from the general population. Journal of Psychosomatic Research, 33: 537-547.

Thirlaway, K. and Benton, D. (1992) Participation in physical activity and cardiovascular fitness have different effects on mental health and mood. Journal of Psychosomatic Research, 36(7), 657-665.

Toskovic, N. (2001) Alterations in selected measures of mood with a single bout of dynamic Taekwondo exercise in college-age students. Perceptual and Motor Skills, 92 (3), 1031-1038. 
Ussher, M., Stanbury, L., Cheeseman, V. \& Faulkner, G. (2007) Physical Activity Preferences and Percieved Barriers to Activity Among Persons with Severe Mental Illness in the United Kingdom. Psychiatric Services, 58, $405-408$

Wardle, J., Steptoe, A., Gulis, G., Sek, H., Todorova, I., Vogele, C., \& Ziarko, M. (2004). Depression perceived control, and life satisfaction in university students from central-Eastern and western Europe. International Journal of Behavioural Medicine, 11 (1):27-36.

Zigmond, A. and Snaith, R.P. (1983) The Hospital Anxiety and Depression Scale. Acta Psychiatrica Scandinavia, 67, 361-370. 
Figure 1 presents the HADS anxiety and depression scores for each physical activity group
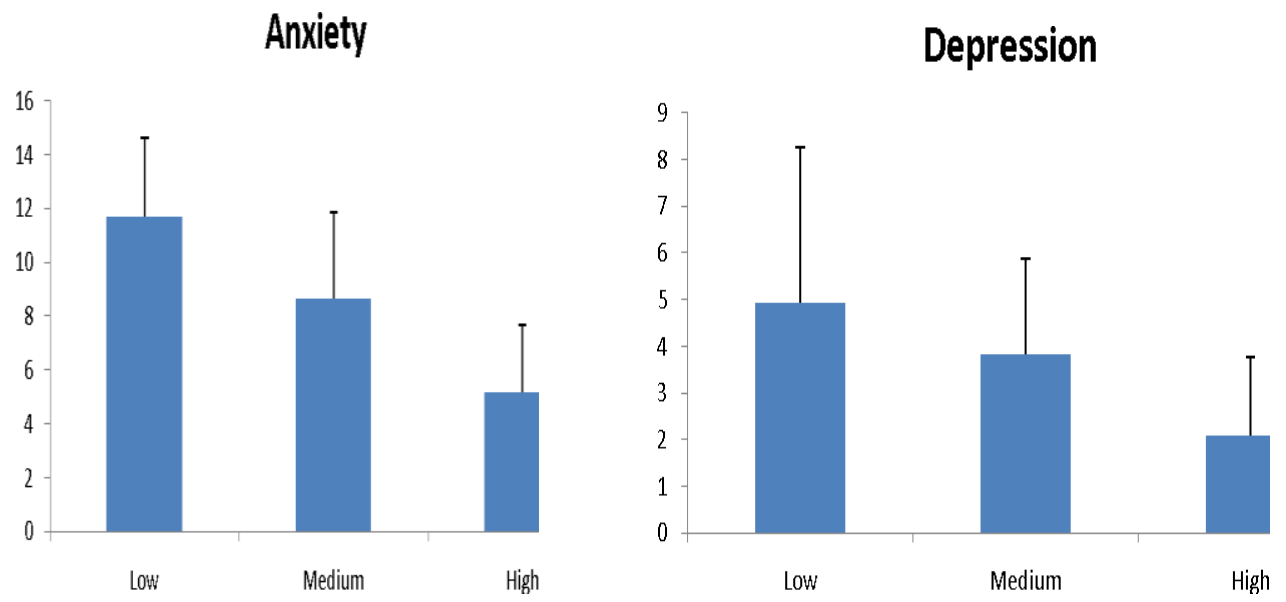
Table 1. Percentage of students falling in each of the severity boundaries of the Anxiety and Depression subscales of the HADS.

\begin{tabular}{|l|c|c|}
\hline & Anxiety & Depression \\
\hline Normal & 40 & 90 \\
\hline Mild & 28 & 8 \\
\hline Moderate & 26 & 2 \\
\hline Severe & 6 & 0 \\
\hline
\end{tabular}


Table 2. Physical Activity score for the low, medium and high groups.

\begin{tabular}{|l|c|c|}
\hline & Physical Activity Score & $95 \%$ Confidence Interval \\
\hline Low physical activity $(\mathrm{N}=34)$ & 3.26 & 0.56 to 5.97 \\
\hline Medium physical activity $(\mathrm{N}=33)$ & 68.45132 & 107.97 to 156.51 \\
\hline High physical activity $(\mathrm{N}=33)$ & 554.94 & 444.48 to 665.40 \\
\hline
\end{tabular}

